\title{
A IMPORTÂNCIA DA ENFERMAGEM DE REABILITAÇÃo \\ Nas Unidades De Cuidados InTEnsivos Portuguesas
}

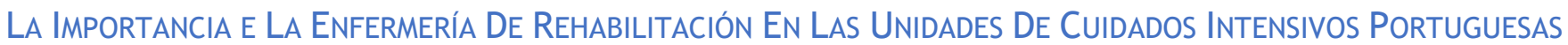 THE IMPORTANCE Of ReHABILITATION NuRsing IN THE PoRTUguese INTENSIVE CARE UNITS}

\author{
Roberto Miguel Gonçalves Mendes ${ }^{1,2}$; Manuel Lourenço Nunes ${ }^{1,2}$ \\ 1 - Faculdade de Ciências da Saúde, Universidade da Beira Interior, Portugal; 2 - Unidade Local de Saúde de Castelo Branco
}

\section{RESUMO}

Objetivo: Conhecer a importância da Enfermagem de Reabilitação nas unidades de cuidados intensivos (UCl's) portuguesas.

Métodos: Análise secundária do inquérito nacional de avaliação da organização dos cuidados de reabilitação, nas unidades de cuidados intensivos portuguesas.

Resultados: Em Portugal há 2.9 enfermeiros por unidade, com formação especializada em reabilitação por unidade. Este valor aumenta nas unidades em que a reabilitação tem por base um modelo misto e diminui nas unidades que recorrem a equipas externas especializadas. $40 \%$ destes profissionais não desempenham funções na sua área de especialização e apenas $26.4 \%$ desempenham funções a tempo inteiro. As unidades com enfermagem de reabilitação a tempo inteiro efetuam mais frequentemente a avaliação da condição física na alta (38.7 versus $8.9 \%$ ), contudo não há participação destes profissionais na avaliação pós-alta em nenhuma UCI. Não são observáveis diferenças nos resultados assistenciais entre as $\mathrm{UCl}$ que integram enfermeiros de reabilitação e as que não o fazem.

Conclusão: Os enfermeiros com especialidade em enfermagem de reabilitação estão presentes na maioria das UCI's nacionais e constituem uma peça importante nos cuidados prestados.

Palavras chave: enfermagem de reabilitação; cuidados intensivos; organização e administração

\section{RESUMEN}

Objetivo: Conocer la importancia de la rehabilitación de enfermería en las unidades de cuidados intensivos (UCl's) portuguesas.

Métodos: Análisis secundaria del estudio nacional para evaluar la organización de la atención de rehabilitación en unidades de cuidados intensivos portuguesas.

Resultados: En cada UCI portuguesa hay 2.9 enfermeras con formación especializada en rehabilitación. Este valor aumenta en las unidades en que la rehabilitación se basa en un modelo mixto y disminuye en las unidades que recurren a equipos externos especializados. $40 \%$ de estos profesionales no desempeñan funciones en su área de especialización y sólo el $26.4 \%$ desempeñan funciones a tiempo completo. Las unidades con enfermería de rehabilitación a tiempo completo efectúan más a menudo la evaluación de la condición física en el alta (38.7 frente al $8.9 \%)$, sin embargo no hay participación de estos profesionales en la evaluación post alta en ninguna UCl. No se observan diferencias en los resultados asistenciales entre las $\mathrm{UCl}$ que integran enfermeros de rehabilitación y las que no lo hacen.

Conclusión: Los enfermeros con especialidad en enfermería de rehabilitación están presentes en la mayoría de las $\mathrm{UCI}$ nacionales y constituyen una pieza importante en los cuidados prestados.

Palavas clave: enfermería en rehabilitación; cuidados críticos; organización y administración

\section{ABSTRACT}

Objective: To know the importance of Rehabilitation Nursing in Portuguese intensive care units (ICUs).

Methods: Secondary analysis of the national evaluation survey of the organization of rehabilitation care in Portuguese intensive care units.

Results: In Portugal there are 2.9 nurses with specialized training in rehabilitation per unit. This value increases in units where rehabilitation is based on a mixed model and decreases in units that resort to specialized external teams. $40 \%$ of these professionals do not perform functions in their area of specialization and only $26.4 \%$ perform full-time functions. Units with full-time rehabilitation nursing more frequently assess the physical condition at discharge (38.7 versus $8.9 \%$ ), however, these professionals do not participate in the post-discharge evaluation in any ICU. There are no observable differences in care outcomes among ICUs that are part of rehabilitation nurses and those who do not.

Conclusion: Nurses with a specialty in rehabilitation nursing are present in most of the national ICUs and are an important part of the care provided.

Keywords: rehabilitation nursing; critical care; organization and administration 


\section{INTRODUÇÃO}

Na maioria dos países desenvolvidos a reabilitação faz parte dos cuidados prestados à pessoa em situação crítica, sendo reconhecida a sua importância em particular na mobilização precoce, uma prática segura e benéfica que deve constituir uma prioridade neste contexto. ${ }^{(1)}$

Por norma, a mobilização e outras técnicas de reabilitação, são asseguradas maioritariamente por fisioterapeutas integrados na equipa ou em regime de chamada, dependendo do país em análise. ${ }^{(2,3)}$ Nas unidades de cuidados intensivos portuguesas a fisioterapia é feita maioritariamente em regime de chamada pelo médico intensivista, contudo no processo de reabilitação intervêm também enfermeiros com formação especializada em reabilitação, vulgarmente designados enfermeiros de reabilitação (ER). ${ }^{(4)}$ Esta especialização da enfermagem surgiu, em Portugal, na década de 60 tendo por base o modelo Norte Americano. Numa fase inicial formaram-se especialistas para o Centro de Medicina Física e Reabilitação do Alcoitão e posteriormente, o aumento do número de ER's permitiu que passassem a integrar serviços de reabilitação ou cinesiterapia respiratória e que integrassem também cuidados de saúde primários e serviços hospitalares, sem que tenha havido uma distribuição homogenia com base nas necessidades destes cuidados especializados. ${ }^{(5)}$

Segundo a Ordem dos Enfermeiros "o enfermeiro especialista em enfermagem de reabilitação concebe, implementa e monitoriza planos de enfermagem de reabilitação diferenciados, baseados nos problemas reais e potenciais das pessoas. (...) A sua intervenção visa (...) assegurar a manutenção das capacidades funcionais dos clientes, prevenir complicações e evitar incapacidades, assim como proporcionar intervenções terapêuticas que visam melhorar as funções residuais, manter ou recuperar a independência nas atividades de vida (...)". O especialista em enfermagem de reabilitação intervém sobretudo "ao nível das funções neurológica, respiratória, cardíaca, ortopédica"(6) enquadrando-se naquilo que serão as necessidades de cuidados de reabilitação do doente crítico. ${ }^{(7-9)}$

Estes enfermeiros estão dispersos por quase todas as unidades, ainda que, por falta de recursos ou opções de gestão, nem sempre desempenhando funções dentro da área de especialização. Ainda assim, podemos encontrar ER's integrados na equipa multidisciplinar das unidades de cuidados intensivos (UCl's) ou integrados em equipas especializadas que se deslocam às unidades para prestar cuidados de reabilitação, fazendo parte de uma organização heterogenia assente em 3 modelos base: ${ }^{(4)}$

1. Modelo interno - cuidados de reabilitação prestados pela equipa da unidade, presente em cerca de $23 \%$ das unidades;

2. Modelo externo - cuidados de reabilitação prestados por equipa externa especializada, presente em $25 \%$ das unidades;

3. Modelo Misto - cuidados de reabilitação prestados pela equipa da unidade em articulação com cuidados por equipa externa, num misto dos modelos anteriores, a situação mais comum que abrange $52 \%$ das unidades.

Neste contexto, O ER está presente em todas as unidades que usam o modelo interno e em cerca de $19 \%$ das equipas externas especializadas que prestam cuidados de reabilitação ao doente critico. ${ }^{(4)}$

Neste artigo pretendemos analisar mais ao pormenor esta informação de forma a compreender a importância da Enfermagem de Reabilitação nas unidades de cuidados intensivos portuguesas. A questão de investigação colocada foi: Qual a importância dos enfermeiros de reabilitação nos cuidados ao doente critico, adulto, em Portugal? São objetivos específicos deste trabalho:

- Determinar a disponibilidade de enfermeiros com formação especializada em reabilitação nas $\mathrm{UCl}$ portuguesas;

- Avaliar a taxa de utilização destes profissionais para o desempenho de funções especializadas na área de reabilitação;

- Avaliar se o número de enfermeiros de reabilitação em cada unidade está relacionado com a organização dos cuidados de reabilitação;

- Determinar a participação dos enfermeiros de reabilitação na mobilização dos doentes críticos;

- Avaliar a influência da presença de enfermeiros de reabilitação nos resultados assistenciais;

- Avaliar a participação dos enfermeiros de reabilitação em possíveis avaliações do doente após a alta da $\mathrm{UCl}$.

\section{MÉTODOS}

Análise secundária do inquérito nacional de avaliação da organização dos cuidados de reabilitação nas unidades de cuidados intensivos portuguesas. Estudo realizado entre novembro de 2016 e março de 2017, através de inquérito on-line aos Enfermeiros Chefes ou Responsáveis das $58 \mathrm{UCl}$ 's de adultos que integram a base de dados da Sociedade Portuguesa de Cuidados Intensivos. 0 inquérito foi composto por um total de 28 questões, agrupadas em: caraterização da instituição, caraterização da unidade, caraterização da equipa, organização dos cuidados de reabilitação, disponibilidade de recursos e resultados. ${ }^{(4)}$

A análise estatística foi realizada através do programa IBM SPSS Statistics versão 22. No que respeita à estatística descritiva foram calculadas frequências, percentagens, médias e desvios padrão.

Foi utilizado o teste de Qui Quadrado para comparar a distribuição do número de ER de acordo com os diversos modelos de organização dos cuidados de reabilitação e para comparar a avaliação da condição física na alta entre UCl's com e sem ER.

A comparação dos resultados assistenciais realizou-se através do teste de Mann-Withney para amostras independentes. 
Utilizou-se um nível de significância de 0.05 .

Estudo aprovado pela Comissão de Ética da Universidade da Beira Interior (Parecer CE-FCS-2016028).

\section{RESULTADOS}

Obtiveram-se 48 inquéritos válidos relativos a 42 unidades médico-cirúrgicas ou polivalentes, 3 unidades neurocirúrgicas e 2 unidades cardiotorácicas (1 inquérito não refere o tipo de unidade). Estas unidades representam um total de 399 camas de cuidados intensivos e 132 camas de cuidados intermédios.

Disponibilidade de enfermeiros com formação em reabilitação

Uma unidade integra na sua equipa, em média, $31.6 \pm$ 14.7 enfermeiros, dos quais cerca de $9.6 \%$ possuem formação especializada em enfermagem de reabilitação. Estas $\mathrm{UCl}^{\prime}$ s incluem um total de 140 enfermeiros com este tipo de especialização, em média, $2.9 \pm 1.8$ enfermeiros por unidade.

- 93.8\% das unidades têm enfermeiros com formação especializada em reabilitação na sua equipa;

- 75.0\% das unidades têm enfermeiro com formação especializada em reabilitação em exercício de funções especializadas;

- 45.8\% das unidades têm enfermeiro com formação especializada em reabilitação em exercício de funções especializadas a tempo inteiro.

Em termos absolutos, $60 \%$ dos enfermeiros com formação especializada desempenham funções especializadas, $26.4 \%$ a tempo inteiro e $33.6 \%$ a tempo parcial (gráfico 1).

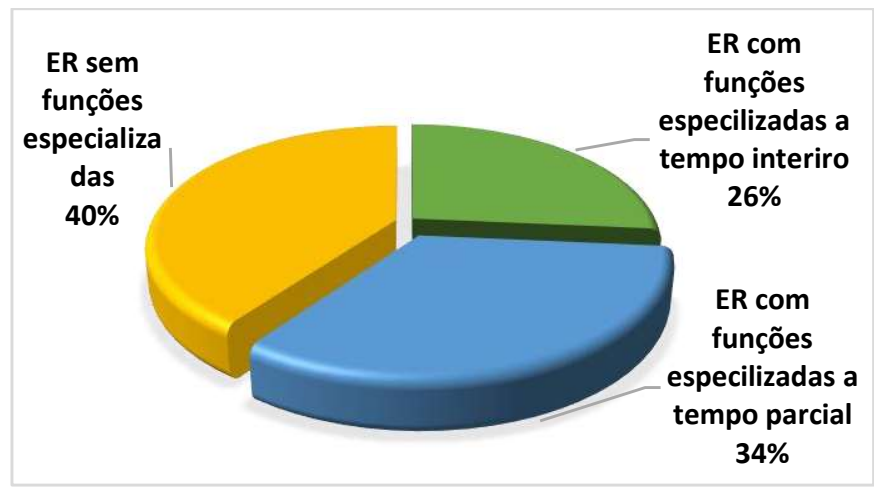

Gráfico 1. Tempo destinado ao exercício de funções especializadas

Analisando a distribuição do número de ER de acordo com os diversos modelos de organização dos cuidados de reabilitação (Tabela 1) verificamos que, em termos médios, é no modelo misto (articulação dos cuidados de reabilitação prestados pela equipa da $\mathrm{UCl}$ com equipas externas especializadas) que existe maior número de enfermeiros com formação especializada em reabilitação por $\mathrm{UCI}$ (3.6 \pm 2.0 enfermeiros/unidade). Em oposição as unidades em que os cuidados de reabilitação são prestados por equipas externas são as que têm menos ER's na sua equipa (1.58 \pm 1.17$)$.
Para além dos ER's integrados nas equipas de cuidados intensivos, em 10 unidades (cerca de 17\%) estes profissionais integram as equipas externas que prestam cuidados de reabilitação nesses serviços.

\section{Participação na mobilização e levante dos doentes}

Para além das atividades de reabilitação realizadas no leito os ER's, independentemente do modelo de organização dos cuidados de reabilitação, são geralmente os dinamizadores da mobilização dos doentes para fora do leito (Gráfico 2) e têm participação ativa nesse procedimento, geralmente em articulação com o enfermeiro cuidador (Gráfico 3).

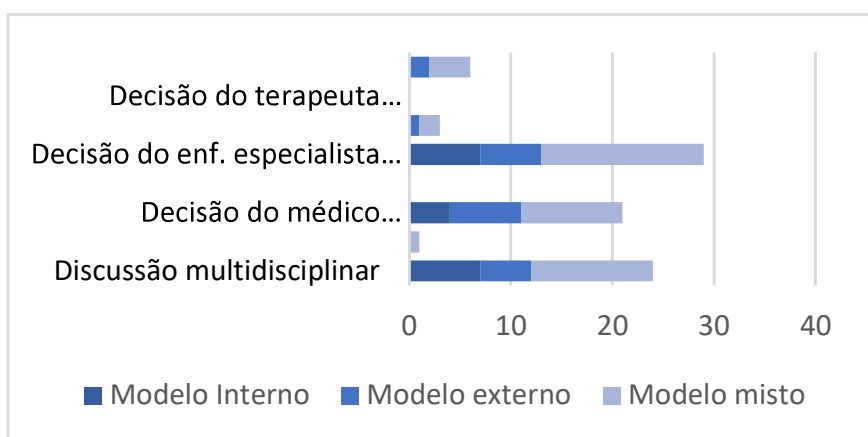

Gráfico 2. Forma como é tomada a decisão de mobilizar os doentes para fora do leito

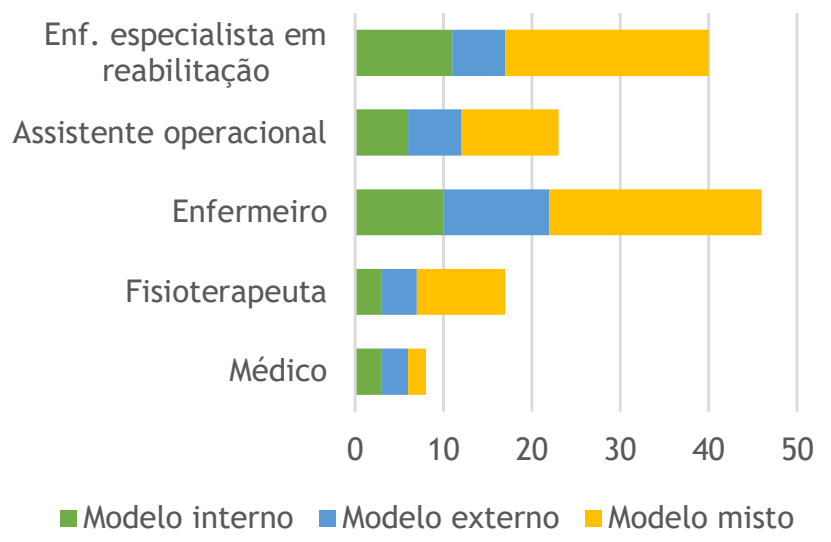

Gráfico 3. Elementos que habitualmente participam na mobilização dos doentes para fora do leito

\section{Influência nos resultados}

Das 48 unidades em análise apenas 19 referiram os resultados relativos a tempo de internamento, tempo de ventilação e taxa de mortalidade. Estes resultados não diferem significativamente por existirem na equipa enfermeiros com formação em reabilitação ou por existirem ER's a tempo inteiro (Tabela 2).

\section{Participação na avaliação à alta e pós-alta}

No que respeita à avaliação na alta, apenas em cerca de $22 \%$ das unidades é avaliada a funcionalidade dos doentes. Contudo observam-se diferenças significativas 


\begin{tabular}{|c|c|c|c|c|c|}
\hline & Modelo de organização & Modelo interno & Modelo externo & Modelo misto & Valor de $p$ \\
\hline \multirow{2}{*}{$\begin{array}{r}\mathrm{N}^{\circ} \text { de enfermeiros } \\
\text { especializados em } \\
\text { reabilitação }\end{array}$} & Soma & 31 & 19 & 90 & \multirow{2}{*}{0.003} \\
\hline & (Média \pm Desvio padrão) & $(2.82 \pm 0.75)$ & $(1.58 \pm 1.17)$ & $(3.60 \pm 2.00)$ & \\
\hline \multirow{2}{*}{$\begin{array}{r}N^{\circ} \text { de enfermeiros de } \\
\text { reabilitação a tempo } \\
\text { inteiro }\end{array}$} & Soma & 14 & 3 & 20 & \multirow{2}{*}{0.096} \\
\hline & (Média \pm Desvio padrão) & $(1.27 \pm 1.42)$ & $(0.25 \pm 0.45)$ & $(0.80 \pm 0.96)$ & \\
\hline \multirow{2}{*}{$\begin{array}{r}\mathrm{N}^{\circ} \text { de enfermeiros de } \\
\text { reabilitação a tempo } \\
\text { parcial }\end{array}$} & Soma & 14 & 0 & 33 & \multirow{2}{*}{0.006} \\
\hline & (Média \pm Desvio padrão) & $(1.27 \pm 1.35)$ & $(0.00 \pm 0.00)$ & $1.32 \pm 1.60)$ & \\
\hline \multirow{2}{*}{$\begin{array}{r}N^{\circ} \text { de enfermeiros de } \\
\text { reabilitação que não } \\
\text { desempenham funções }\end{array}$} & Soma & 3 & 16 & 37 & \multirow{2}{*}{0.129} \\
\hline & (Média \pm Desvio padrão) & $(0.27 \pm 0.46)$ & $(1.33 \pm 1.37)$ & $(1.48 \pm 2.20)$ & \\
\hline
\end{tabular}

Tabela 1. Análise da distribuição do número de ER de acordo com os diversos modelos de organização dos cuidados de reabilitação

\begin{tabular}{|c|c|c|c|c|}
\hline \multicolumn{2}{|l|}{ UCI com enfermeiro de reabilitação } & Não & Sim & Valor de $\mathrm{p}$ \\
\hline & $\mathrm{N}$ & 2 & 26 & \multirow{2}{*}{0.894} \\
\hline & Média \pm Desvio padrão & $7.45 \pm 0.78$ & $7.37 \pm 2.33$ & \\
\hline \multirow{2}{*}{$\begin{array}{l}\text { Tempo médio de ventilação invasiva } \\
\text { (dias) }\end{array}$} & $\mathrm{N}$ & 0 & 20 & \\
\hline & Média \pm Desvio padrão & & $5.73 \pm 2.69$ & \\
\hline \multirow{2}{*}{ Taxa de mortalidade } & $\mathrm{N}$ & 1 & 23 & \multirow{2}{*}{1.000} \\
\hline & Média \pm Desvio padrão & $21.00 \pm \ldots$ & $20.79 \pm 6.21$ & \\
\hline \multicolumn{2}{|c|}{ UCI com enfermeiro de reabilitação a tempo inteiro } & Não & Sim & Valor de $\mathrm{p}$ \\
\hline \multirow{2}{*}{ Tempo médio de internamento (dias) } & $\mathrm{N}$ & 16 & 12 & \multirow{2}{*}{0.347} \\
\hline & Média \pm Desvio padrão & $7.65 \pm 2.23$ & $7.02 \pm 2.32$ & \\
\hline \multirow{2}{*}{$\begin{array}{l}\text { Tempo médio de ventilação invasiva } \\
\text { (dias) }\end{array}$} & $\mathrm{N}$ & 11 & 9 & \multirow{2}{*}{0.370} \\
\hline & Média \pm Desvio padrão & $6.43 \pm 2.24$ & $4.87 \pm 3.07$ & \\
\hline \multirow{2}{*}{ Taxa de mortalidade } & $\mathrm{N}$ & 13 & 11 & \multirow{2}{*}{0.228} \\
\hline & Média \pm Desvio padrão & $19.51 \pm 4.95$ & $22.32 \pm 7.12$ & \\
\hline
\end{tabular}

Tabela 2. Comparação dos resultados assistenciais entre unidades com e sem enfermeiros de reabilitação

entre as unidades que têm ER a tempo inteiro e as que não possuem enfermeiros nessas condições $\left(X^{2}{ }_{(2)}=\right.$ 5.373; $p=0.020 ; N=41$ ), com as primeiras a realizarem mais frequentemente esse tipo de avaliação $(38,7$ versus $8,9 \%$ ).

Os números de avaliação após alta são ligeiramente inferiores, apenas com $12.5 \%$ das unidades a referirem efetuar este tipo de acompanhamento e, ainda assim, de forma muito díspar. Não foi referida a participação de enfermeiros especialistas em reabilitação nesta avaliação.

\section{DISCUSSÃO}

Os enfermeiros com especialização em enfermagem de reabilitação estão presentes na maior parte das $\mathrm{UCI}$ a nível nacional, uma situação sem paralelo a nível internacional. (10) Para além do trabalho que desenvolvem junto dos doentes, tomando a iniciativa de iniciar precocemente o processo de reabilitação e elaborando um plano adequado à condição do doente, (4) estes profissionais promovem, como se evidencia nesta análise, a mobilização dos doentes para fora do leito, indo de encontro às orientações que preconizam a precocidade na mobilização destes doentes. ${ }^{(11)}$

A par do trabalho clínico são desenvolvidos projetos de investigação em áreas concretas da reabilitação dos doentes críticos, nomeadamente a nível respiratório, ${ }^{(12-16)}$ motor ${ }^{(17)}$ e também na promoção da autonomia ${ }^{(18)}$ e nas competências para o atendimento à pessoa com dependência no autocuidado ${ }^{(19)}$. Este desenvolvimento parece estar intimamente relacionado com a criação dos cursos de mestrado em enfermagem de reabilitação 
uma vez que a maior parte das investigações foram desenvolvidas nesse âmbito.

Apesar de neste artigo se apresentarem alguns traços do que é a intervenção da enfermagem de reabilitação nas unidades de cuidados intensivos, estamos convictos que apenas apresentamos a ponta do iceberg e que a investigação nesta área tem elevado potencial. Seria interessante conhecer de facto o quão precoce é mobilização e se cumprimos as recomendações internacionais, o que nem sempre se tem verificado nos trabalhos de investigação desenvolvidos em outros países. ${ }^{(20-22)}$ Neste aspeto em particular, a enfermagem de reabilitação poderia reforçar a sua posição, pois trata-se de um contexto sensível em que é fundamental o domínio de um conjunto de competências muito especificas ao alcance dos ER's. Esta afirmação passa também pela documentação da prática e os indicadores são a melhor ferramenta. A Ordem dos Enfermeiros e o Colégio da Especialidade de Enfermagem de Reabilitação já deram alguns passos nesse sentido, divulgando um conjunto de indicadores potencialmente sensíveis aos cuidados de reabilitação. ${ }^{(23)}$ Há que identificar aqueles que mais se adequam e eventualmente complementar com alguns indicadores mais específicos deste contexto. (24-26) Para desenvolver este trabalho será importante aumentar a participação dos ER na avaliação da condição física dos doentes na alta e no pós alta e rentabilizar os $40 \%$ de enfermeiros com formação especializada que à data não desempenhavam funções na área.

\section{CONCLUSÃO}

Os enfermeiros com especialidade em enfermagem de reabilitação estão presentes na maioria das UCl's nacionais e constituem um elemento importante nos cuidados ao doente crítico, situação ímpar no contexto internacional. A sua intervenção passa por desenvolver planos de cuidados individuais, incluindo mobilização para fora do leito, e a sua atividade é sustentada por uma crescente produção científica. Não possuímos neste momento dados que nos permitam inferir sobre a influência nos resultados assistenciais, de um modo geral, ou mais especificamente na funcionalidade ou em ganhos no autocuidado, alvos da intervenção desta especialidade.

\section{REFERÊNCIAS BIBLIOGRÁFICAS}

1. Stiller K. Physiotherapy in intensive care: An updated systematic review. Chest. 2013;144(3):825-47.

2. Bakhru R, Wiebe D, McWilliams D, Spuhler V, Schweickert W. An environmental scan for early mobilization practices in U.S. ICUs. Crit Care Med . 2015;43(11):2360-9.

3. Taito S, Sanui M, Yasuda H, Shime N, Lefor AK. Current rehabilitation practices in intensive care units: a preliminary survey by the Japanese Society of Education for Physicians and Trainees in Intensive Care (JSEPTIC) Clinical Trial Group. J Intensive Care. 2016;4(66): 3p.

4. Mendes R, Nunes L, Pinho JA, Gonçalves R. Organização dos cuidados de reabilitação nas unidades de cuidados intensivos portuguesas. Rev Bras Ter Intensiva. 2018;30(1):57-63.

5. Castanheira F. História da enfermagem de Reabilitação. Revista da Ordem dos Enfermeiros. 2003;(3):12-3.

6. Ordem dos Enfermeiros. Regulamento das competências específicas do enfermeiro especialista em enfermagem de reabilitação. [s.l.], Ordem dos Enfermeiros. 2010: 5p.

7. Malone $D$, Ridgeway $K$, Nordon-Craft a., Moss $P$, Schenkman $M$, Moss $M$. Physical therapist practice in the intensive care unit: results of a national survey. Phys Ther. 2015;95(10):1335-44.

8. Gosselink R, Bott J, Johnson M, Dean E, Nava S, Norrenberg M, et al. Physiotherapy for adult patients with critical illness: recommendations of the European Respiratory Society and European Society of Intensive Care Medicine Task Force on Physiotherapy for Critically III Patients. 208;34(7):1188-99.

9. França EÉT de, Ferrari F, Fernandes P, Cavalcanti R, Duarte A, Martinez BP, et al. Fisioterapia em pacientes críticos adultos: recomendações do Departamento de Fisioterapia da Associação de Medicina Intensiva Brasileira. Rev Bras Ter Intensiva. 2012;24(1):6-22.

10. Schoeller S, Martins M, Ribeiro I, Lima D, Padilha M, Gomes B. Breve panorama mundial da enfermagem de reabilitação. Rev Port Enferm Reabil. 2018;(0):7-13.

11. Hodgson CL, Stiller K, Needham DM, Tipping CJ, Harrold M, Baldwin $C E$, et al. Expert consensus and recommendations on safety criteria for active mobilization of mechanically ventilated critically ill adults. Crit Care. 2014;18(6):658.

12. Marreiros P. Intervenção do enfermeiro de reabilitação na prevenção de complicações resporatórias na pessoa submetida a ventilação invasiva [em linha]. Relatório de estágio do Curso de Mestrado em Enfermagem, Área de Especialização de Enfermagem de Reabilitação. Escola Superior de Enfermagem de Lisboa, Lisboa. 2015. 249 p.

13. Simões J. Intervenções do enfermeiro especialista em enfermagem de reabilitação à pessoa submetida a ventilação mecânica invasiva. Dissertação de Mestrado em Enfermagem de Reabilitação. Escola Superior de Enfermagem de Lisboa, Lisboa. 2016. $191 \mathrm{p}$.

14. Silva MJ. Intervenção do enfermeiro especialista em enfermagem de reabilitação, em contexto de cuidados intensivos, á Pessoa com doença pulmonar obstrutiva drónica submetida a ventilação não invasiva. Relatório de estágio do Curso de Mestrado em Enfermagem, Área de Especialização de Enfermagem de Reabilitação. Escola Superior de Enfermagem de Lisboa, Lisboa. 2012. $153 \mathrm{p}$.

15. Gonçalves C. Intervenção do Enfermeiro Especialista em Enfermagem de Reabilitação na pessoa em situação de atelectasia em cuidados intensivos. Relatório de estágio do Curso de Mestrado em Enfermagem, Área de Especialização de Enfermagem de Reabilitação. Escola Superior de Enfermagem de Lisboa, Lisboa. 2016. 297 p.

16. China M. Avaliação do impacto da intervenção do enfermeiro de reabilitação com a utilização da reeducação funcional respiratória no doente crítico, numa unidade de cuidados intensivos. Dissertação de Mestrado em Enfermagem de Reabilitação. Escola Superior de Enfermagem de Coimbra, Coimbra. 2015. 130 p.

17. Santos AR, Oliveira IS De, Silveira T. Mobilização precoce em UCI. Rev Ciências da Saúde da ESSCVP. 2010;(2):19-24.

18. Romão C. Contributos da enfermagem de reabilitação no incremento da autonomia do utente em cuidados intensivos. Instituto Politécnico de Portalegre, Portalegre. 2015. 62 p.

19. Pereira JA. Competências do enfermeiro de reabilitação com doentes dependentes no autocuidado em cuidados intensivos. Dissertação de Mestrado em Enfermagem de Reabilitação Escola Superior de Enfermagem do Porto, Porto. 2012. 168 p.

20. Berney SC, Harrold M, Webb SA, Seppelt I, Patman S, Thomas PJ, et al. Intensive care unit mobility practices in Australia and New Zealand: a point prevalence study. Crit Care Resusc. Australia; 2012;15(4):260-5.

21. Pires-Neto RC, Lima NP, Cardim GM, Park M, Denehy L, Camargo Pires-Neto R, et al. Early mobilization practice in a single Brazilian intensive care unit. J Crit Care. 2015;30(5):896-900.

22. Sibilla A, Nydahl P, Greco N, Mungo G, Ott N, Unger I, et al. Mobilization of mechanically ventilated patients in Switzerland. J Intensive Care Med. 2017;20(10).

23. Ordem dos Enfermeiros. Enfermagem de Reabilitacao: Bilhetes de identidade dos indicadores que integram o core de indicadores por categoria de enunciados descritivos dos padrões de qualidade dos cuidados de enfermagem de reabilitação. [s.l.], Ordem dos 
Enfermeiros. 2018. 335p.

24. Scottish Intensive Care, Society Audit Group. Minimum standards and quality Indicators for critical care in Scotland, Version 3.0 [em linha]. Acedido a 17/07/18, em: https: //www.scottishintensivecare.org.uk/quality-

improvement/quality-indicators-SICSAG/

25. Sociedad española de medicina intensiva crítica y unidades coronarias. Quality indicators in critically ill patients, 2107 update. [s.l.], Sociedad española de medicina intensiva crítica y unidades coronarias. 2017. 198p.

26. Luna EC, Quiroga IC. Diseño de indicadores para el cuidado respiratorio y movilización temprana en una unidad de cuidado intensivo. Rev cienc salud. 2014;12(1):47-62. 